\title{
STUDY OF THE OXYGEN ELECTRODE REACTION USING MIXED CONDUCTING OXIDE SURFACE LAYERS. PART II: SMALL SIGNAL ANALYSIS
}

\author{
M.P. VAN DIJK* $*$ K.J. DE VRIES * and A.J. BURGGRAAF \\ Twente University of Technology, Department of Chemical Engineering, \\ Laboratory for Inorganic Materials Science, \\ P.O. Box 217, 7500 AE Enschede, The Netherlands
}

Received 23 December 1985; accepted for publication 6 March 1986

The oxygen gas electrode has been studied for a number of mixed conducting oxide surface layers on top of $\mathrm{Gd}_{2} \mathrm{Zr}_{2} \mathrm{O}_{7}$ ( $\mathrm{TGZO}$ ) solid electrolytes. In part II of this paper we present the results of frequency dispersion measurements for the electrode reaction, supplying additional information to the results of current-overvoltage experiments presented in part $I$. For both kinds of experiments the same trends were observed for the electrode polarization. Best results are obtained for a surface layer of TGZO, while p-type mixed conducting oxides give less decreased values of the electrode polarization. High electrode capacitances were found in the case of mixed conducting surface layers (about $700 \mathrm{~F} / \mathrm{m}^{2}$ ). The electrode reactions follow a Butler-Volmer type of equation. Most probably a diffusion process is rate controling the overall charge transfer process.

\section{INTRODUCTION}

In Part I of this paper we presented the preparation and characterization of layers of mixed conducting oxides on solid electrolytes prepared by radio frequency sputtering. Materials used were the solid electrolyte $\mathrm{Gd}_{2} \mathrm{Zr}_{2} \mathrm{O}_{7}$ (TGZO) and the mixed conducting oxides $\mathrm{Tb}_{2} \mathrm{Zr}_{2} \mathrm{O}_{7+\mathrm{y}}(\mathrm{TGZ100})$ and $\mathrm{Ce}_{0 .} \mathrm{Tb}_{0.3^{\mathrm{O}} \mathrm{O}_{2-\mathrm{y}}}$ (CT30). Carrying out current-overvoitage experiments for the oxygen gas electrode reaction, it was determined that the presence and nature of a sputtered layer on top of a solid electrolyte has a large effect on the polarization behaviour. With a sputtered layer of the ionic conducting TGZO material the highest current densities were obtained compared to the current densities for the bare solid electrolyte at the same overvoltage values. Mixed, p-type, conducting TGZ100 and CT30 surface layers gave a smaller increase of the current densities. In addition to the current-overvoltage experiments the electrode surface layers are characterized in this part of the paper by small signal complex impedance analysis, ylelding information about the electrode resistance $\left(R_{e l}\right)$ :

* present address: Koninklijke Shell Laboratori um Amsterdam, P.O. Box 3003, 1003 AA Amsterdam, The Netherlands * to whom correspondence is to be sent

$$
R_{e l}=\left(\frac{n}{1}\right)_{n=0}=\frac{R T}{\left(\alpha_{a}{ }^{+\alpha_{c}}\right) F I_{0}}
$$

and the capacitive behaviour of the electrode interface. Results of the frequency dispersion measurements, according to the experimental procedure outlined in part I of this paper, are split up in a resistive part and a capacitive part for convenience.

\section{RESULTS}

\subsection{Resistive part}

Values of the electrode resistance $\left(R_{e l}\right)$ were obtained from the complex impedance diagrams as the difference of the intercept values of the polarization curve with the real axis at 1 ow and high frequencies.

Fig. 1 shows the electrode resistances as a function of temperature at $\mathrm{P}_{\mathrm{O}_{2}}=0.21$ atm (scaled values acoording the procedure described in the appendix of part I). Activation enthalpies are listed in Table I. Just as in the $1-n$ experiments the configuration with the TGZO sputtered layer has the lowest electrode polarization followed by CT 30 and TGZ100. It is not excluded that at higher temperatures than covered in our experiments the CT30 layer turns out to have the lowest resistance. The bare solid electro-

0 167-2738/86/\$03.50 @ Elsevier Science Publishers B.V. (North-Holland Physics Publishing Division) 


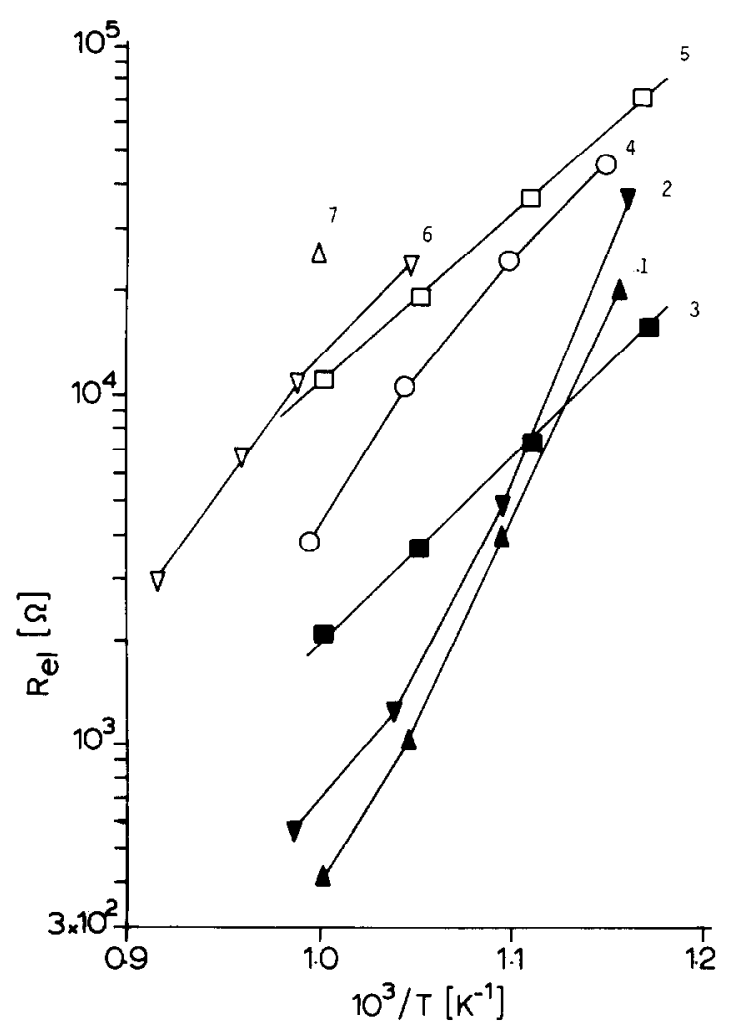

Fig.1. Scaled electrode resistances $\left(R_{e l}\right)$ as a function of temperature at $\mathrm{P}_{\mathrm{O}_{2}}=0.21 \mathrm{~atm}$.

1: $600 \mathrm{~nm} \mathrm{CT30/Pt}$

3: $600 \mathrm{~nm} \mathrm{TGZO/Au}$ 2: $600 \mathrm{~nm} \mathrm{TGZO/Pt}$

5: $150 \mathrm{~nm} \mathrm{TGZ100/ \textrm {Au }}$ 7: bare TGZO

lyte shows the highest resistance.

All electrode configurations with the Austrip show a similar activation enthalpy of about $100 \mathrm{~kJ} / \mathrm{mol}$, except for the $150 \mathrm{~nm}$ and $600 \mathrm{~nm}$ CT30 layers, where the activation enthalpy increases for higher temperatures. For the $600 \mathrm{~nm}$ TGZO and $150 \mathrm{~nm}$ CT3O it was found that the activation enthalpies were independent of $\mathrm{P}_{\mathrm{O}_{2}}$. The activation enthalpy for the bare solid electrolyte is somewhat 1 arger.

Evidently the configurations with the Ptstrip perform better in the high temperature range. Towards lower temperatures the curves for Pt and Au approach, which is an interesting point for pumping applications.

For $\mathrm{Pt}$ hardly any difference in $\mathrm{R}_{\mathrm{el}}$ is noticed whether the sputtered layer consists of TGZO or CT30. The curves of $R_{e l}$ versus $T$ show a similar bend, with a high value of the activa-
TABLE I. Activation enthalpies for $R_{\text {el }}$ for the various electrode configurations. In cases where no Arrhenius behaviour was observed for $R_{e l}$ the numbers in the column for $\Delta H$ describe the gradual transition in the slope of the curves. The first number shows the low temperature limit, the second shows the high temperature $l i m i t$.

\begin{tabular}{llrr}
\hline $\begin{array}{l}\text { electrode } \\
\text { configuration }\end{array}$ & strip & $\begin{array}{c}\Delta \mathrm{H} \\
\text { (kJ/mol) }\end{array}$ & $\begin{array}{c}\mathrm{P}_{\mathrm{O}_{2}} \\
\text { (atm) }\end{array}$ \\
\hline & & & \\
bare TGZ0 & $\mathrm{Au \#}$ & 120 & 0.21 \\
$600 \mathrm{~nm}$ TGZ0 & $\mathrm{Au}$ & 101 & 0.01 \\
$"$ & $\mathrm{Au}$ & 106 & 0.21 \\
$"$ & $\mathrm{Au}$ & 110 & 0.43 \\
$"$ & $\mathrm{Pt}$ & $240-140$ & 0.21 \\
$150 \mathrm{~nm} \mathrm{CT30}$ & $\mathrm{Au}$ & $100-150$ & 0.21 \\
$600 \mathrm{~nm}$ CT30 & $\mathrm{Au}$ & $100-150$ & 0.21 \\
& $\mathrm{Pt}$ & $240-140$ & 0.21 \\
$150 \mathrm{~nm} \mathrm{TGZ100}$ & $\mathrm{Au}$ & 99 & 0.21 \\
\hline
\end{tabular}

* For this configuration a symmetrical two electrode cell was used with Au strips to carry out temperature dependent frequency dispersion measurements. Consequently these $R_{\text {el }}$ results cannot be compared quantitatively with the other results which were scaled. However it is allowed to compare $\Delta H$ values.

tion enthalpy at the lower temperatures changing to a lower value at the higher temperatures. This indicates a change in rate determining step, as becomes clear from the $\mathrm{P}_{2}$ dependence of $\mathrm{R}_{e l}$. Apparently the influence of $\mathrm{Pt}$ on the electrode reaction process is large and obscures possible influences of the electrolyte surface layer.

F18. 2 shows the $\mathrm{P}_{\mathrm{O}_{2}}$ dependence of $\mathrm{R}_{\mathrm{el}}$. It is seen that for all configurations with a Au-strip the lines run parallel. $\mathrm{R}_{\mathrm{el}}$ varies as $\mathrm{P}_{0} \mathrm{~m}$, with $-0.45<\mathrm{m}<-0.50( \pm 0.05$ for each line). In the case of the bare solid electrolyte sample $m=-0.42( \pm 0.05)$ and $f$ or the Pt-strip on $600 \mathrm{~nm}$ CT $30, \mathrm{~m}=-0.34( \pm 0.05)$. In the latter case $m$ changes to a positive value around $\mathrm{P}_{2}=0.1 \mathrm{~atm}$. This was found too by other investigators for Pt-electrodes (e.g. $1,2)$ and it can be related with the fraction of occupied adsorption sites. At high temperatures $\theta<1 / 2(\theta=$ fraction of occupied surface sites) and at low temperatures $\theta>$ $1 / 2$. The fact that the $\mathrm{P}_{\mathrm{O}_{2}}$ value at which the sign of m changes, decreases for decreasing temperature, Implies that the activation enthalpy for the electrode reaction (measured at $\mathrm{P}_{\mathrm{O}_{2}}=0.1 \mathrm{~atm}$ ) in this temperature range 


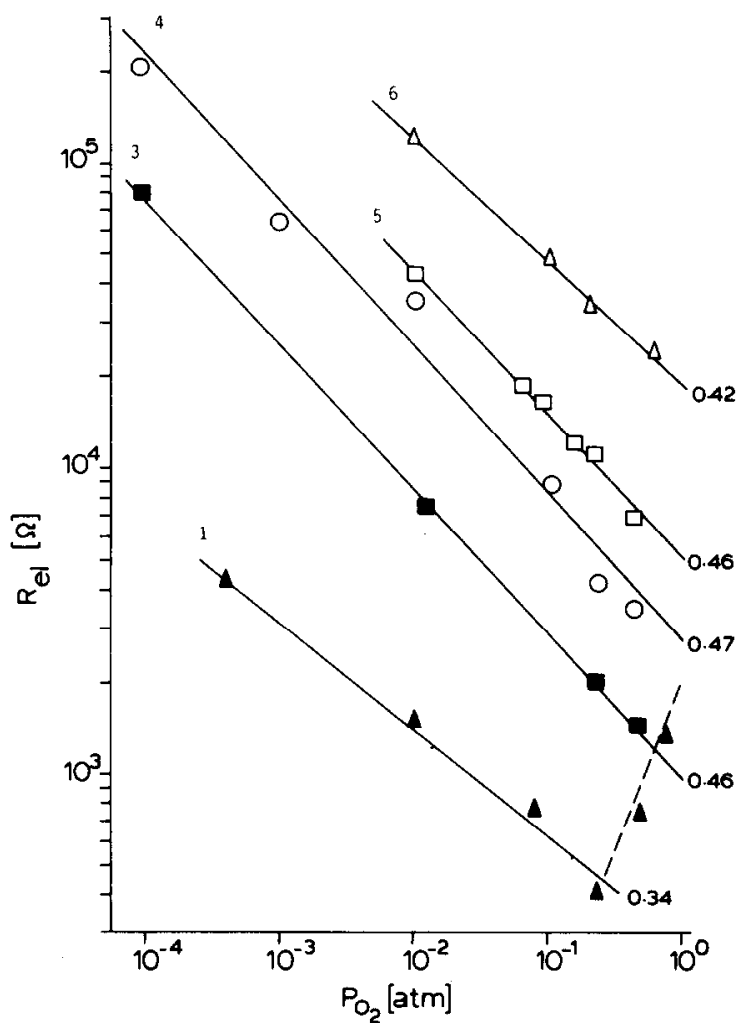

Fig.2. Scaled electrode resistances as a function of $\mathrm{P}_{2}$ at $\mathrm{T}=727^{\circ} \mathrm{C}$.
1: $600 \mathrm{~nm}$ CT $30 / \mathrm{Pt}$
3: $600 \mathrm{~nm} \mathrm{TGZO/Au}$
4: $600 \mathrm{~nm} \mathrm{CT30/Au}$
5: $150 \mathrm{~nm} \mathrm{TGZ100/Au}$
6: bare TGZO

covers a change in the mechanism. For the configurations with the Au-strip no such change in sign of $m$ was observed, which implies that $\theta<1 / 2$ in the whole $\mathrm{P}_{\mathrm{O}_{2}}$ range investigated.

\subsection{Capacitive behaviour}

Figs.3a-f show the complex impedance diagrams for the various electrode configurations investigated. The inset of most $f$ igures shows the high frequency part of the polarization arcs on an enlarged scale. Numbers along the arcs indicate ${ }^{10} \mathrm{log}$ (frequency) values.

The polarization arcs generally consist of a relatively large semicircle towards the lower frequencies. In the case of electrode conflgurations with a $\mathrm{Pt}-\mathrm{strip}$ and in the case of the $600 \mathrm{~nm}$ sputtered TGZ0 layer with a Au-strip, the semicircles show rather 1 arge depression angles (up to $35^{\circ}$ ) and are sometimes skewed. For the mixed conducting oxides CT30 and TGZ100 sputtered layers with a Au-strip the semicircle depression angles are relatively small (10$\left.15^{\circ}\right)$.

Towards the high frequencies in most cases a straight I ine behaviour is observed, the behavlour of which is independent of $\mathrm{P}_{2}$ (inset of the figures). The bare solid electrolyte with the Au strip shows a polarization consisting of two partially separated arcs. The low frequency arc has a rather low semicircle depression angle $\left(=10^{\circ}\right)$.

The impedance data were interpreted in terms of tentative equivalent electrical circuits. Circuit parameters were obtained using a non linear least squares ref inement program (3). For simplicity the constant phase element (CPE) is used in the refinement procedures:

$$
Y(C P E)=Y_{0}(j \omega)^{n}
$$

With $n=1$ this element represents a capacitance and for $n=0$ a resistor. For $n=0.50$ the CPE equals a classical Warburg impedance (2) in a semi-infinite medi um:

$Z(W)=\sqrt{ } 2 K_{W}(j w)^{-1 / 2}$ or $Y(W)=\left(\sqrt{ } 2 K_{W}\right)^{-1}(j w)^{1 / 2}$

The Warburg impedance describes a diffusion process and takes a different form for particular boundary conditions $(1,4)$. The complete expression for a $R$-type Warburg impedance $(R-W)$ is:

$Y(R-W)=Y_{0}(R-W) \times B(1+j) \times \operatorname{cotanh}[B(1+j)]$

where $B=\delta \gamma \frac{W}{2 D}, Y_{0}(R-W)=\frac{n^{2} F^{2} C D}{R T}, \delta$ is a characteristic diffusion distance, $C$ the equilibrium concentration, $D$ the diffusion coefficient and $\omega$ the angular frequency $2 \pi f$. In the limit $\delta \gg \gamma \frac{2 D}{w}, 1 . e$. In the case where the diffusion medium can be considered sem1- infinite, [3] reduces to [2]. The Warburg constant, consequently, is defined by:

$$
K_{W}=\frac{R T}{n^{2} F^{2} C \sqrt{2 D}}
$$

The low frequency part of the impedance diagrams is discussed first. Depressed semicircles can be analyzed with a resistance ( $R$ ) and a $C P E$ in parallel (the depression angle equals $n * \frac{\pi}{2}$ ). Small semiclrcle depression is usually attributed to a relaxation process with a spread in relaxation times, for instance described by a Cole-Cole distribution function around $\omega_{0}=(R C)$ average $(5,6)$. Using the CPE and $R$ parallel circuif, the average yalue of $C$ is obtalned from $(R C)$ average $=\left(Y_{0} R\right)^{1 / n}$. This 


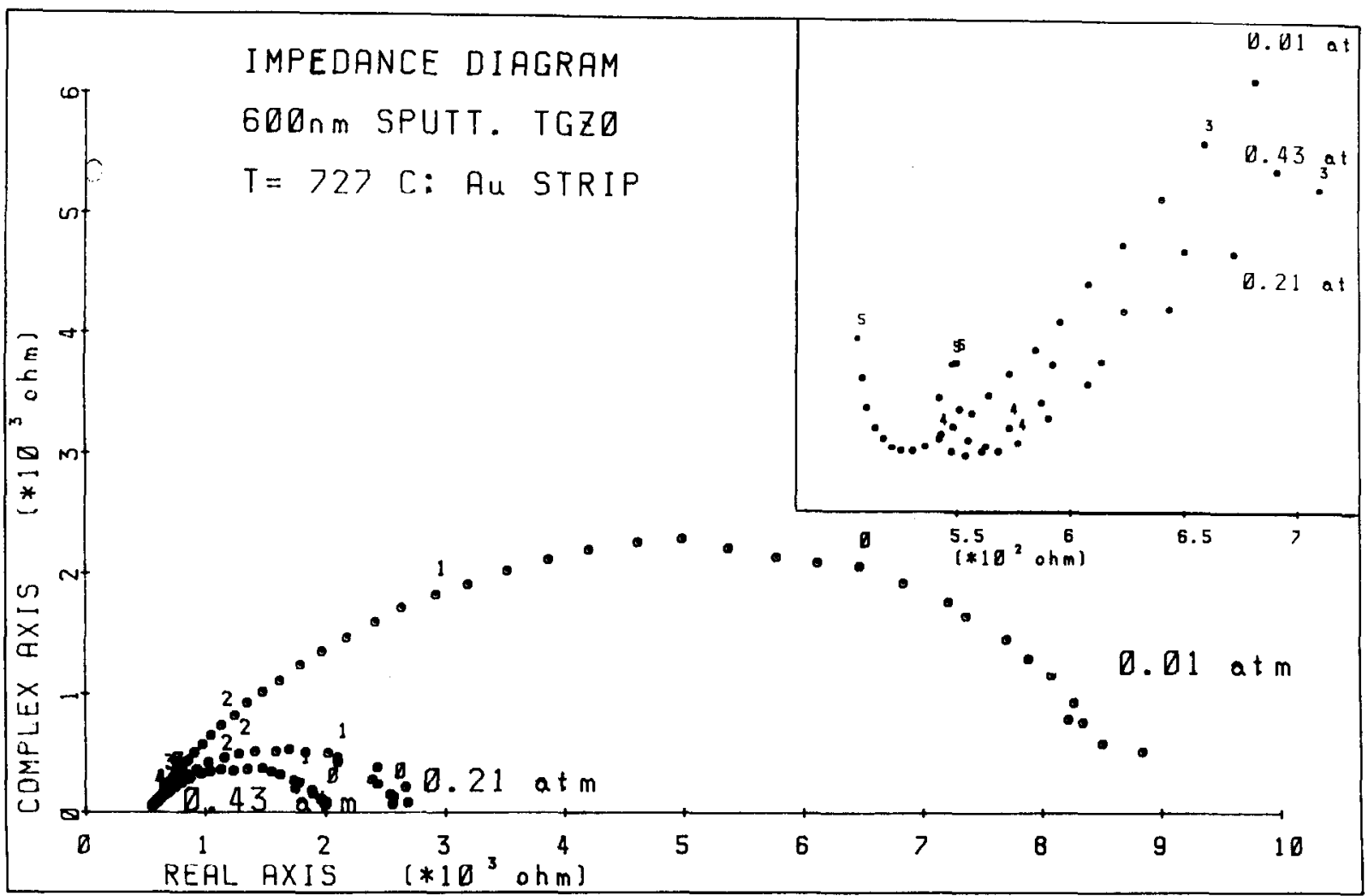

Fig.3a Polarization curves for a $600 \mathrm{~nm}$ sputtered layer of TCZO at varying $\mathrm{P}_{2}$.

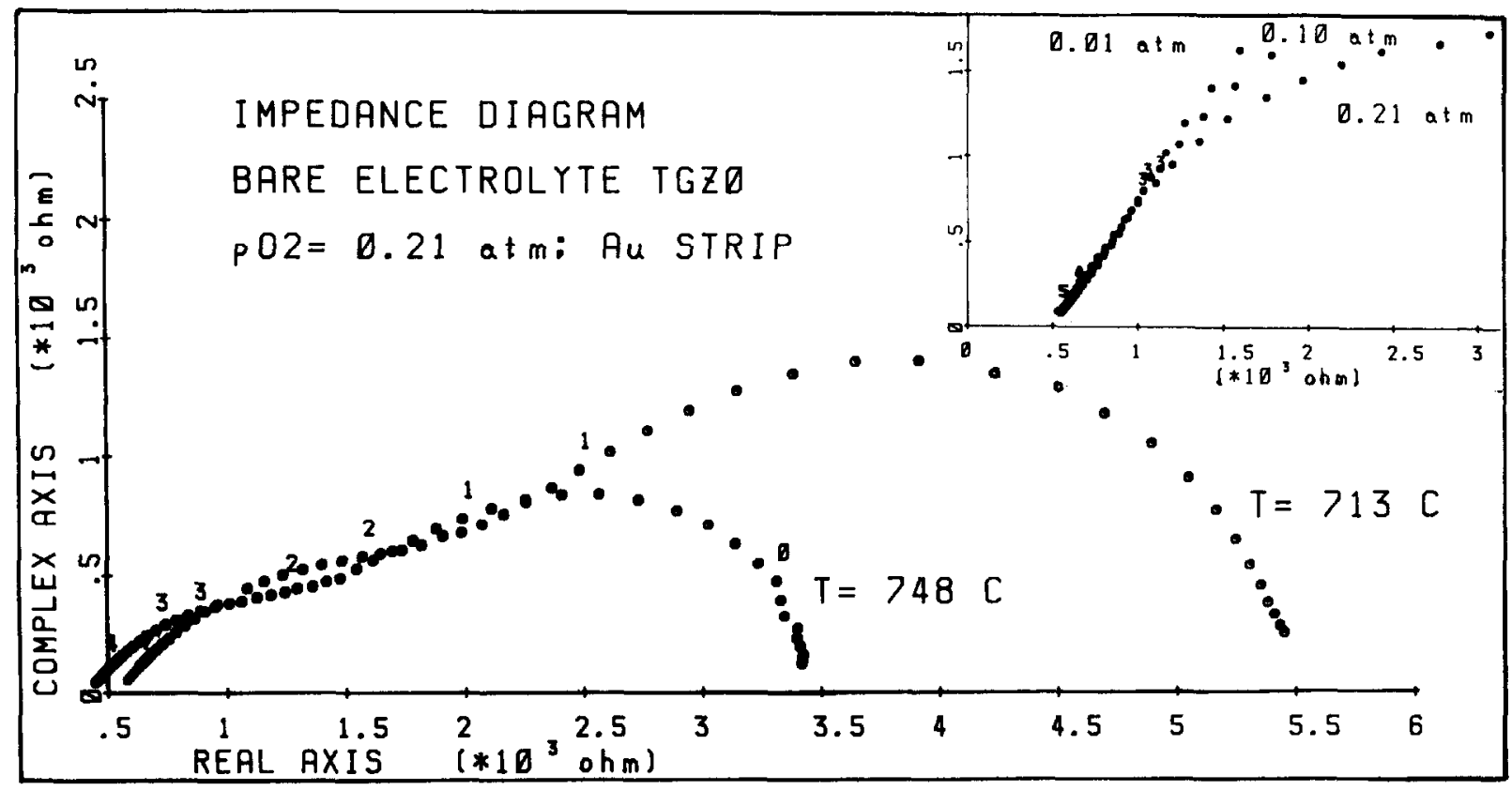

Fig.3b Polarization curves for the bare solid electrolyte sample (see footnote Table I) 


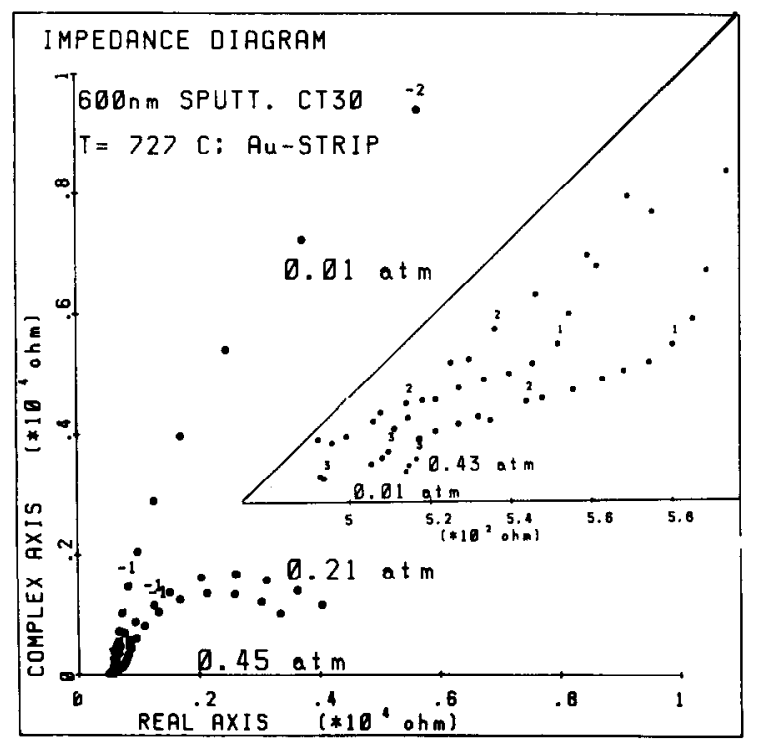

Fig. 3c Polarization curves for a $600 \mathrm{~nm}$ sputtered layer of CT 30 .

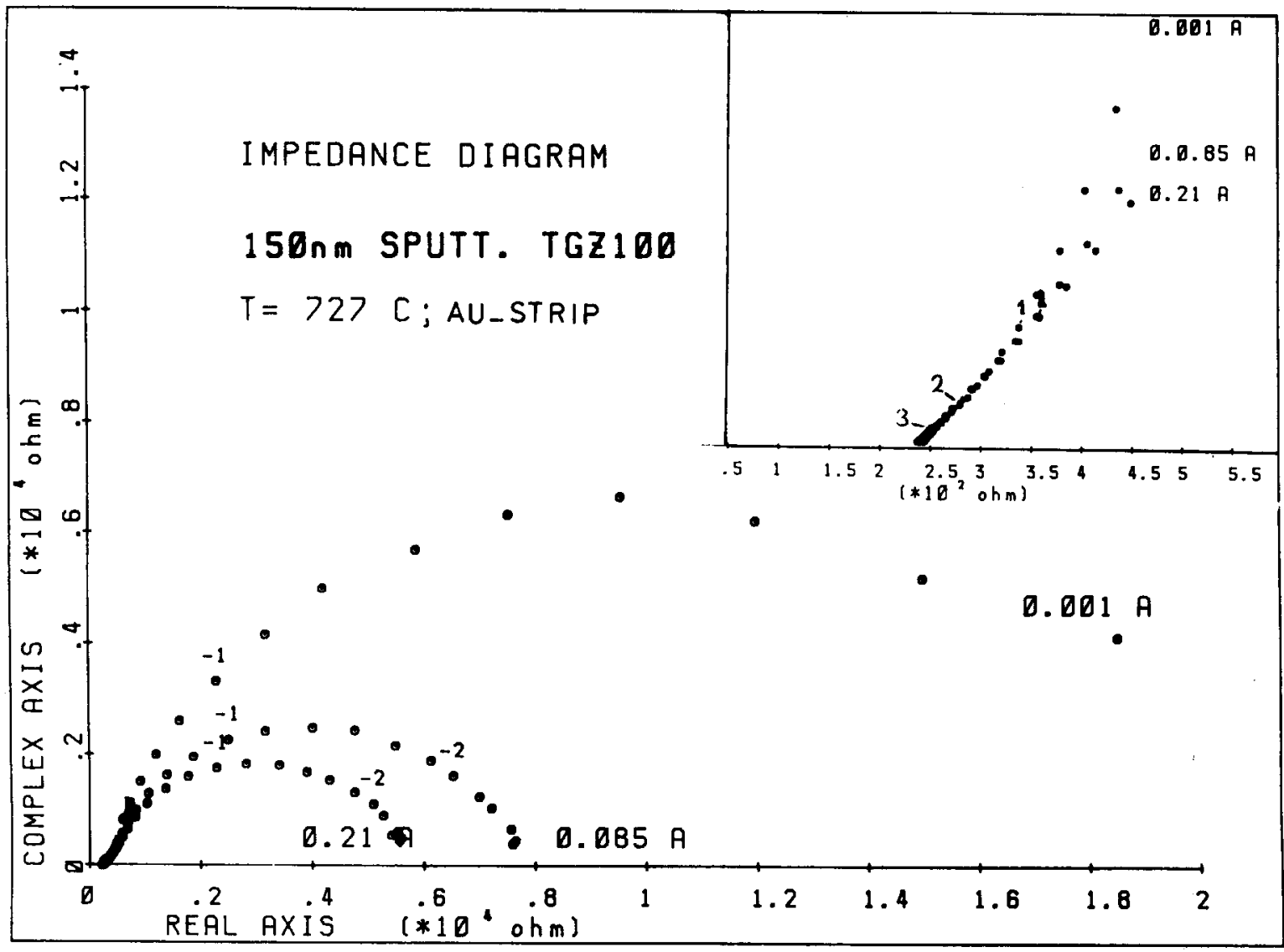

Fig.3d Polarization curves for a $150 \mathrm{~nm}$ sputtered layer of TGZ100. 


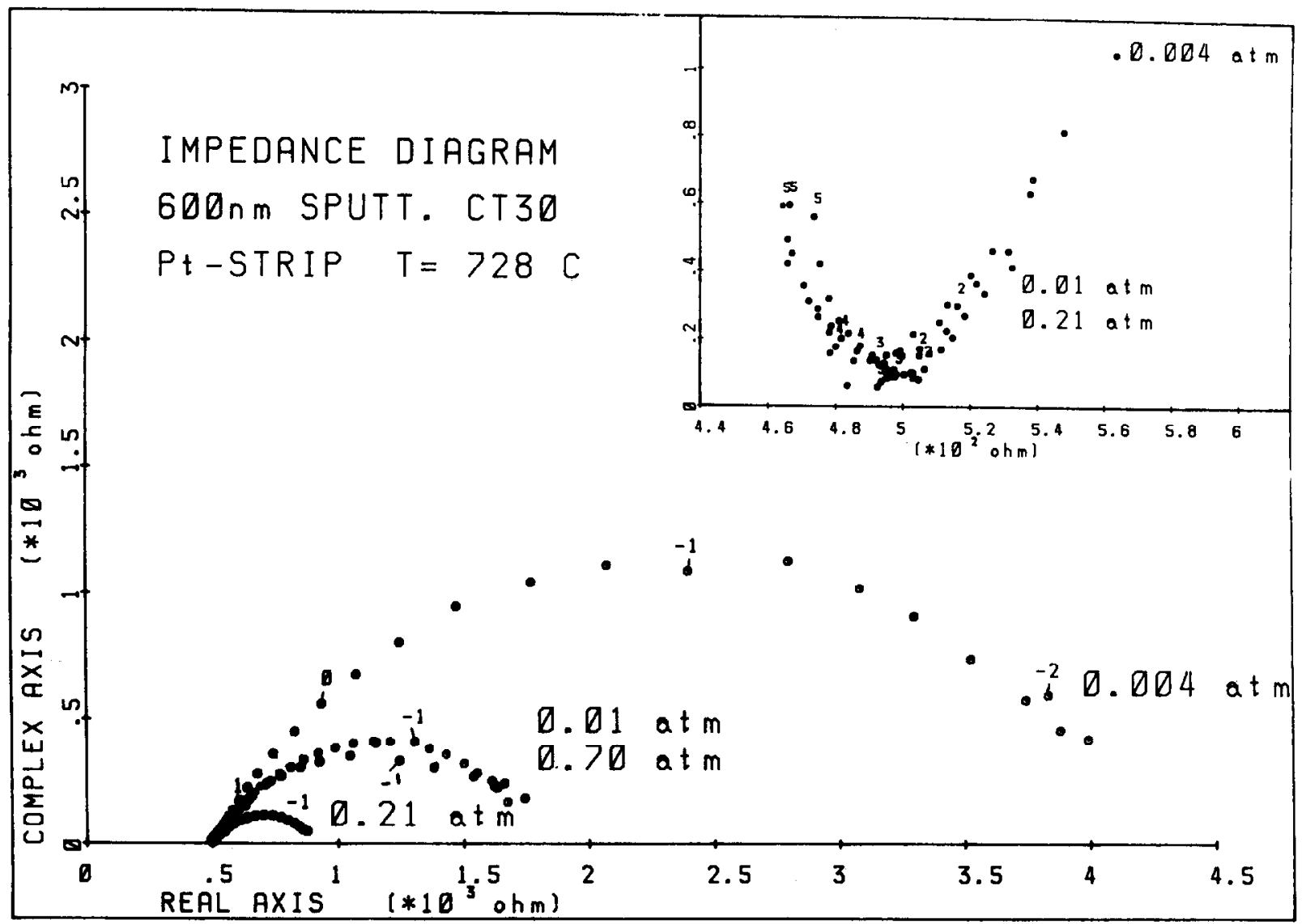

Fig.3e Polarization curves for a $600 \mathrm{~nm}$ sputtered layer of CT30 and Pt-strip contacts.

IMPEDANCE DIAGRAM

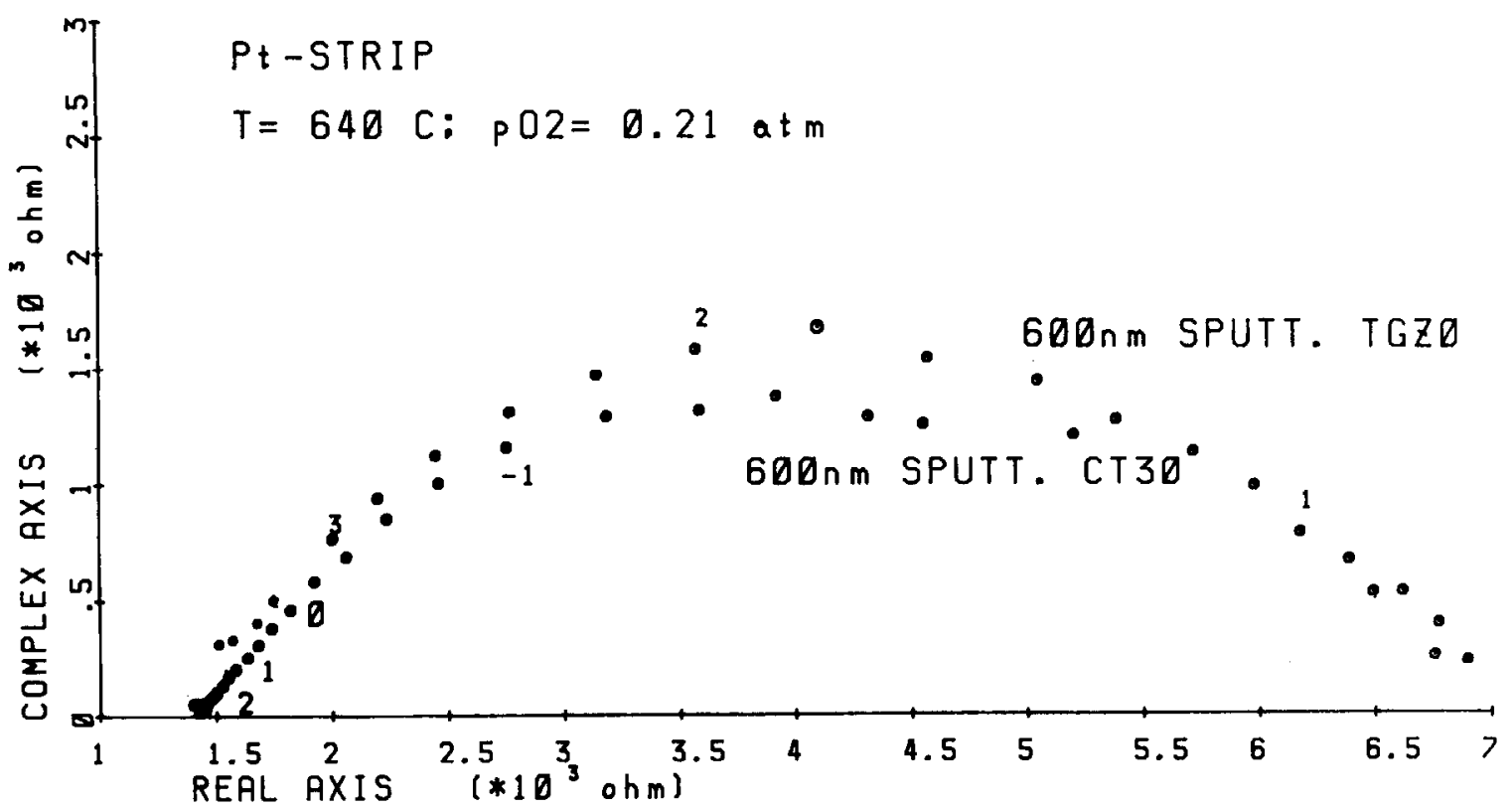

F1g.3f Polàrization curves for TGZO and CT30 sputtered layers with Pt-strip contacts. Note the different frequency ranges. 
TABLE II.

Fitted resuits for the low frequency part of the 1mpedance dlagrams at $\mathrm{T}-727^{\circ} \mathrm{C}$ and $\mathrm{P}_{\mathrm{O}_{2}}=0.21$ atm ( $R$ and CPE in parallel).

\begin{tabular}{|c|c|c|c|c|c|}
\hline \multirow[t]{2}{*}{ Configuration } & \multirow[t]{2}{*}{ Strip } & \multirow{2}{*}{$\begin{array}{l}\mathrm{R} \\
(\Omega)\end{array}$} & \multicolumn{2}{|c|}{$\mathrm{CPE}$} & \multirow{2}{*}{$\begin{array}{l}{ }^{n} \mathrm{n} \\
(\mathrm{F})\end{array}$} \\
\hline & & & $Y_{0}$ & $\mathrm{n}$ & \\
\hline $\begin{array}{l}\text { bare - TGZ0 } \\
600 \mathrm{~nm} \text { TGZ0 } \\
600 \mathrm{~nm} \text { CT30 } \\
150 \mathrm{~nm} \text { CT30 } \\
150 \mathrm{~nm} \text { TGZ100 } \\
600 \mathrm{~nm} \text { TGZ0 } \\
600 \mathrm{~nm} \mathrm{CT30}\end{array}$ & $\begin{array}{l}\mathrm{Au} \\
\mathrm{Au} \\
\mathrm{Au} \\
\mathrm{Au} \\
\mathrm{Au} \\
\mathrm{Pt} \\
\mathrm{Pt}\end{array}$ & $\begin{array}{l}3.4 \times 10^{4} \\
2.2 \times 10^{3} \\
4.1 \times 10^{3} \\
1.5 \times 10^{4} \\
7.3 \times 10^{3} \\
6.0 \times 10^{2} \\
4.1 \times 10^{2}\end{array}$ & $\begin{array}{l}1.3 \times 10^{-8} \\
1.7 \times 10^{-3} \\
7.4 \times 10^{-4} \\
2.0 \times 10^{-4} \\
4.2 \times 10^{-4} \\
6.6 \times 10^{-6} \\
9.5 \times 10^{-4}\end{array}$ & $\begin{array}{l}0.89 \\
0.58 \\
0.90 \\
0.85 \\
0.87 \\
0.58 \\
0.65\end{array}$ & $\begin{array}{l}8.7 \times 10^{-7} \\
1.6 \times 10^{-4} \\
8.5 \times 10^{-4} \\
3.8 \times 10^{-4} \\
5.0 \times 10^{-4} \\
1.2 \times 10^{-7} \\
5.8 \times 10^{-4}\end{array}$ \\
\hline
\end{tabular}

procedure was followed for the polarization arcs with small senlcircle depression angle.

Semicircles with rather large depression angles may result from the contribution of a diffusion process to the electrode reaction( 1 ). For instance a Warburg element (W) In parallel to a resistance results in a semicircle with depression angle $45^{\circ}$. An alternative way of interpreting large depression angles can be effected by using circuits of branched ladder networks. Scheider postulates that the capacitive behaviour of the electrode polarization is totally ascribed to charge spreading in the diffuse double layer and a frequency dependent capacitance resulting from the microscopic surface roughness (7). Such a situation is likely to occur for the noble metal strip-solid electrolyte or sputtered layer contact.

For comparison the capacitive value was also calculated in the case of the impedance diagrans with large semicircle depression angles using the CPE description.

Values of the calculated capacities are given in Table II and Figs. 4 and 5 (scaled values). Obviously large differences are found between the solid electrolyte surfaces of TGZO on the one hand and the mixed conducting surface layers of CT 30 and TGZ100 on the other. The type of electrode strip (Pt or $\mathrm{Au}$ ) has no significant influence on the high $\mathrm{C}$-values determined for CT 30 and TG2100. The C-values for a $150 \mathrm{~nm}$ sputtered layer are perhaps scmewhat lower than for a $600 \mathrm{~nm}$ sputtered layer. The temperature influence seems to be small, but an increasing $\mathrm{P}_{2}$ leads to higher $\mathrm{C}$-values in case of TGZ100 and CT30 and lower C-values in case of TGZO.

At the high frequency side of the impedance diagrams straight lines are observed in many cases. For the TGZO bare specimen and for the TGZO sputtered layer with the Au strip this line is observed at relatively large frequencies $\left(10^{4}-10^{3} \mathrm{~Hz}\right)$, while for the Tb-contalning sputtered layers, both with $\mathrm{Au}$ and Pt strips the frequency range is about $10^{3}-10^{2} \mathrm{~Hz}$. This points to considerably larger relaxation times for the predominant process in the case of mixed conducting oxides. It is noticed that

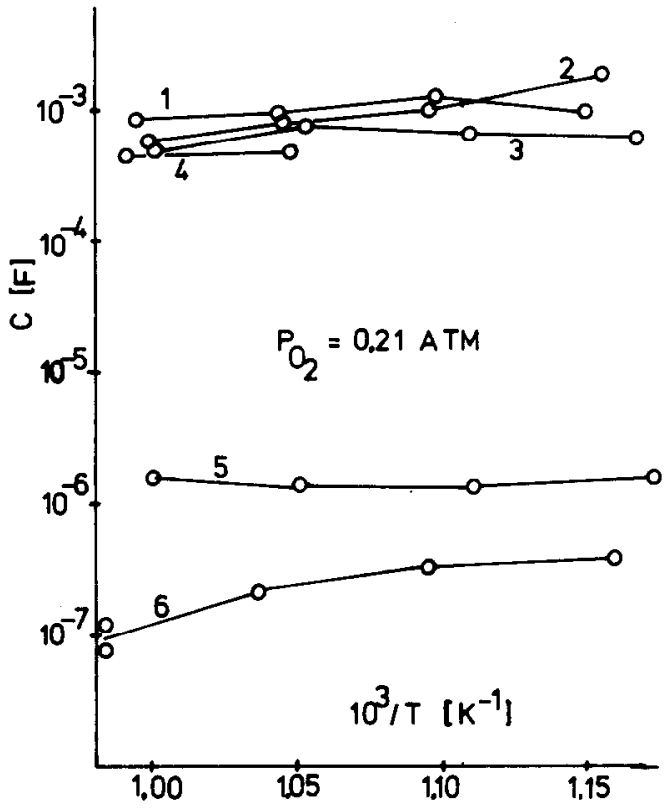

Fig.4. Scaled electrode capacitance as a function of temperature.

1: $600 \mathrm{~nm} \mathrm{CT30/Au} \mathrm{2:} 600 \mathrm{~nm} \mathrm{CT30/Pt}$

3: $150 \mathrm{~nm} \mathrm{TGZ100/Au} \mathrm{4:} 150 \mathrm{~nm} \mathrm{CT30/Au}$

5: $600 \mathrm{~nm} \mathrm{TGZ0/Au} \mathrm{6:} 600 \mathrm{~nm} \mathrm{TGZO/Pt}$ 


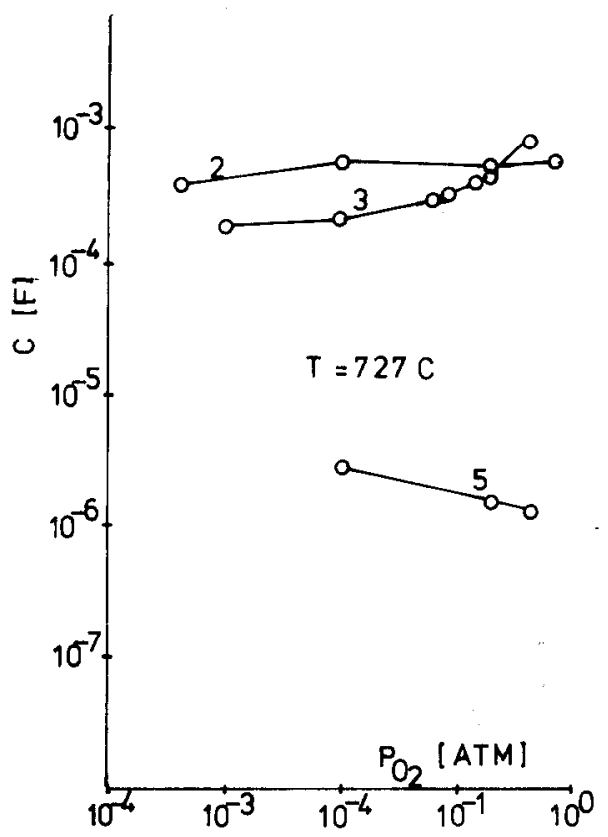

Fig.5. Scaled electrode capacitance as a function of $\mathrm{P}_{2}$.

2: $600 \mathrm{~nm} \mathrm{CT30/Pt}$

5: $600 \mathrm{~nm} \mathrm{TGZO/Au}$

3: $150 \mathrm{~nm} \mathrm{TGZ100/Au}$

this part of the impedance behaviour is independent of $\mathrm{P}_{2}$.

From the impedance data for these high frequency ranges $Y_{0}$ and $n$ can be calculated, using the CPE description. For CT30 and TGZ100 it results that $Y_{0}$ is thermally activated with $\Delta \mathrm{H}=40 \mathrm{~kJ} / \mathrm{mol}$, whl ch is also the value for the activation of $K_{W}$, when $n=0.50$.

For the bare TGZO specimen two rather separated arcs were observed (Fig. 3b). A complete description in terms of an equivalent circuit involves two parts in series, each part consisting of a CPE and a resistance in parallel. The high frequency part has $n=0.50$ and the low frequency part has $n=0.90$ ). It is noted that the high frequency part is rather $\mathrm{P}_{2}$ independent and that $\mathrm{K}_{\mathrm{W}}^{2}$ (and hence $\mathrm{C}^{2} \times \mathrm{D}$ ) and the parallel resistance are activated with $\Delta H$ * $90 \mathrm{~kJ} / \mathrm{mol}$, Indicative of an electrolyte related process.

\section{DISCUSSION,}

\subsection{Au strip electrodes}

All layer electrode configurations with a $\mathrm{Au}$ strip showed similar behaviour in the electrode reaction, w1th respect to the values of $m$ and $\Delta \mathrm{H}$ at low temperature, while at $\mathrm{T}=1000 \mathrm{~K}$ values of $\alpha_{a}^{*} \frac{3}{2}, \alpha_{c}^{*}=\frac{1}{2}$ and $m=\frac{-1}{2}$ were obtained. This indicates that the reaction mechanism shows similarities for all electrode configurations, irrespective of the presence of mixed conductivity in the surface layer of the electrolyte phase.

In principle both a reaction mechanism with charge transfer being rate determining and one with mass transport Iimitation can be considered. Considering charge transfer limitation, the $f$ act that $\alpha_{a}{ }^{*}$ and $\alpha_{c}{ }^{*}$ are not equal indicates as ymmetry in the sequence of reaction steps. For a large number of possible mechanisms, concerning both dissociatively and associatively adsorbed oxygen species, and concerning situations in which different numbers of electrons are being transferred at the same time, values of $\alpha$ and $m$ can be predlcted $(2,8)$. No consistent mechanism with pure charge transfer being rate determining was found which was able to desoribe the present results.

Models including diffusion limitation in the rate determining process of the electrode reaction have been studied in the literature too. Wang and Nowick presented a model in which surface diffusion of atomic oxygen on noble metal electrodes was coupled to a charge transfer reaction step (10). The model predicts $\alpha_{a}^{*}=\frac{3}{2}$, $\alpha_{c}{ }^{*}=\frac{1}{2}$ and $m=\frac{-3}{8}$, if diffusion limitation dominates, while the true Faradaic coefficients $\left(\alpha_{c}=\alpha_{a}=1\right)$ and $m=\frac{-1}{4}$ are found for charge transfer limitation. This model describes experimental results of these authors for porous $\mathrm{Au}$ and $\mathrm{Ag}$, and $\mathrm{Pt}-\mathrm{foll}$ electrodes on doped ceria solid electrolytes $\left(500-800^{\circ} \mathrm{C}\right.$, $\left.10^{-5}-1 \mathrm{~atm}\right)$. We used a modifled model of this type.

An analogous model was very recently described by Nguyen et al. (9). These authors, investigating So-stabilized zirconia with Aupaste electrodes, showed a model in which surface diffusion of $\mathrm{O}_{2}{ }^{-}$species is rate controlling the electrochemical step. It was argued by these authors that adsorption and surface diffusion of oxygen is more likely to occur on the solid electrolyte phase than on the Au particles. The electrochemical reaction sites are connected to regions where the porous Au electrode and the solid electrolyte phase are in contact with each other.

With this model, it was deduced that if diffusion Iimitation dominates, the apparent charge, transfer coefficients $\left(\alpha_{a}^{*}=\frac{3}{2}\right.$ and $\alpha_{c}{ }^{*}=\frac{1}{2}$ ) deviate from the real Faradaic coef ${ } i-$ cients $\left(\alpha_{a}=\alpha_{c}=1\right)$ and that $m=\frac{-1}{2}$. On the other hand, if charge transfer limitation dominates, the apparent charge transfer coefficients became equal to the real coefficients 
and $m=\frac{-1}{4}$. For almost all their experiments Nguyen et al. found that diffusion limitation dominated with an activation enthalpy of $97 \mathrm{~kJ} / \mathrm{mol}$, which agrees very well with values found in our study.

Models of the cited type, which combine diffusion limitation and charge transfer limitation, well explain our experimental results. At the higher temperatures diffusion limitation seems to dominate and the current-overvoltage data can be fitţed to $i=1_{0, \operatorname{app}}\left[\exp \left(\frac{3}{2} n^{*}\right)-\exp \left(-\frac{1}{2} n^{*}\right)\right]$. Here 1 is a function of the parameter $I_{0} / Q$ and $Q$ lsp dimensionless parameter that is a measure of the relative ratio of the charge transfer exchange rate and the diffusion rate. $Q$ always includes a characteristic distance along which diffusion takes place $(l)$, and the product of the diffusion coefficient (D) times the concentration of oxygen species at the adsorption site $\left(C_{0}\right)$. In the model presented by wang and Nowigk

$Q=\left(\frac{i_{0} l^{2}}{2 \operatorname{eDC}_{0}}\right)^{1 / 2}(10)$. At lower temperatures the slight decrease of $\alpha_{a}{ }^{*}$ suggests that charge transfer Iimitation becomes more important.

At $\mathrm{P}_{2}<10^{-2}$ atm $1-n$ curves were observed that showed properties characteristic for limiting currents according to eq. [3] and [4] in part I. This confirms that diffusion I Imitation is at least partially present at higher values of $\mathrm{P}_{\mathrm{O}_{2}}$.

No clear indication of the type of mechanism can be obtained from the value of the activation enthalpy $(* 100 \mathrm{~kJ} / \mathrm{mol})$ since similar values are reported in the literature for both diffusion limited and charge transfer limited processes $(1,9,10)$.

The type of oxide surface layer has a pronounced effect on the relative positions of the $i-n$ curves, as was observed in our experiments. Since values of $\alpha_{a^{*}}$ and $\alpha_{c^{*}}$ are comparable for the various sputtered oxide layers, the difference in the electrode polarizations can only originate from the fact that both charge transfer and diffusion rates are different to a similar extent. The diffusion flux is determined by the product $D \times C_{0}$ and the effects of $D$ and $C_{0}$ cannot be separated simply. Thus a TGZO sputtered layer has the best properties for adsorption $\left(C_{0}\right)$ and/or surface diffusion (D), and the TGZo bare sol 1 d electrolyte has the worst. Incorporation of $\mathrm{Tb}$ or mixed ( $\mathrm{p}-$ type) conductivity negatively influences the properties of $C_{0}$ and/or $D$. These results point to the fact that surface diffusion along the solid electrolyte or sputtered layer phase rather than surface diffusion along the Austrip contact prevails. This is in agreement with the $f$ act that $m=-0.50$ was found, in correspondence with the model of Nguyen et al. which assumes solid electrolyte surface diffusion to prevail. (9).

In order to explain why sputterd layers perform better than the bare solid electrolyte sample, it is interesting to refer now to the results of the catalytic co-oxidation experiments on TGZ powders (11). These results showed that oxides which had been given a heat treatment at $1100-1300^{\circ} \mathrm{C}$ were most active. This corresponds to the heat treatment at $1100^{\circ} \mathrm{C}$ given to the freshly sputtered oxide surface layers. On the other hand it was shown in the present study that preferential orientation occurs within the grains of the sputtered layer. The effect of orientation is not known at present, but in analogy to known effects for adsorption processes on metals, it may have a pronounced effect on adsorption in this case.

The differences in electrode performance between the $\mathrm{Tb}$-containing sputtered layers and the TGZO sputtered layer can result from differences in adsorptive capacity too. It was noticed $(11,12)$ that for Tb-containing oxides the reoxidation step in the catalytic oxidation of $\mathrm{CO}$ was rate determining. This step involves the uptake of oxygen and the donation of electrons, similar to the cathodic reaction described here. It is suggested that electron donation is hindered by the p-type character of the CT 30 and TGZ100 surface layers, in which electron-holes are the mobile charge carriers. Hence it is worth to continue investigations of $\mathrm{O}_{2}$ redox reactions with $n$-type mixed conducting oxides too. The stronger decrease of the electrode resistance at higher temperatures for CT30 compared to TG2100 possibly results from the fact that Ce-containing oxides tend to become $n$-type semiconductors at higher temperatures, annihilating the p-type behaviour of CT30 due to the presence of TD.

The above mentioned results do not imply that p-type materials should not be useful in anodic oxidation reactions of other reactants (e.g. Co, methanol, etc.)

\subsection{Pt strip electrodes}

Irrespective whether a sputtered layer of CT30 or TGZO was applied, the electrode resistance and 1 ts temperature dependence for the electrode combinations with a Pt strip are different from the combinations with Au. The similar behaviour of the CT30 and TGZO sputtered layer combinations, in the case of $\mathrm{Pt}$, suggests that the rate determining step in the electrode process is closely related to the properties of the Pt-strip part of the electrode.

The mechanism can be adequately described by assuming that charge transfer limitation and atomic diffusion limitation on the Pt-metal 
cooperate. This results from the fact that the values of $\alpha_{a}^{*}, \alpha_{c}{ }^{*}$ and $m$ correspond with intermediate values as given by Wang and Nowick (10). From our experiments $\alpha_{a}{ }^{*}=1.1, \alpha_{c}{ }^{*} \approx 0.7$ and $m=-0.34( \pm 0.05)$, which falls in between the values $\alpha_{a}=\alpha_{c}=1.0$ and $\mathrm{m}=-\frac{1}{4}$ for charge transfer 1 imitation and $\alpha_{a} * \frac{3}{2}$, $a_{c}{ }^{*}=\frac{1}{2}$ and $m=-\frac{3}{8}$ for diffusion I imitation. The impedance diagrams correspond with the polarization behaviour found for Pt gauze electrodes by Verkerk et al. (1).

\section{3 Electrode capacitance}

It is supposed that the electrode capacitance, presented in Fig. 4 and Fig.5, corresponds with the double layer capacitance in the case of the ionic conducting TGzo electrode configurations. Taking a mean value of $10^{-6} \mathrm{~F}$ and the surface area of the $A u$ and Pt strip $\left(15 \mathrm{~mm}^{2}\right)$ the double layer capacitance should equal $7 \times 10^{-2} \mathrm{~F} / \mathrm{m}^{2}$. This is quite a small value compared with literature data ranging from $0.3-5 \mathrm{~F} / \mathrm{m}^{2}(1,10)$. Consequent $1 \mathrm{y}$, it is concluded that actually only $2-20 \%$ of the strip surface is effective in the electrode reaction. It should be remembered that this holds for scaled values of the electrode resistance. A similar conclusion can be drawn from the experiment in which two gold strips were used on both sides of a bare specimen in a two electrode impedance measurement. Now the measured electrolyte resistance can be compared with specific conductivity data for TGZO (13) from which an effective contact surface of $\approx 10 \%$ of the geometrical one can be estimated.

Using the value of the geometrical contact surface $\left(15 \mathrm{~mm}^{2}\right)$ a minimum yalue of the electrode capacitance of $70 \mathrm{~F} / \mathrm{m}^{2}$ is obtained in the case of CT30 and TGZ100 sputtered surface layers. Using $10 \%$ as an average value of effective surface area contact, the capacitance is $700 \mathrm{~F} / \mathrm{m}^{2}$. Clearly this value is too large to result from the double layer capacitance, connected to oxygen vacancy redistribution in the outmost surface layers. The capacitance will rather be a pseudocapacitance, which is frequency dependent.

It is supposed that this very high capacitive contribution results from electronic charge injection at the electrode strip contact, which is not compensated immediately by oxygen transport. Effectively, this implies that the stoichiometry of the mixed conducting oxide layers is changed for very low frequencies. This effect also explains the hysteresis observed in the $i-\eta$ curves.

The value of $C$ can be related to the number of electron holes present. In (13) it was shown that $\left[\mathrm{Tb}^{4+}\right]$ and the electronic conductivity decrease in parallel, when the $\mathrm{P}_{2}$ is

decreased. The value of $C$ also decreases for

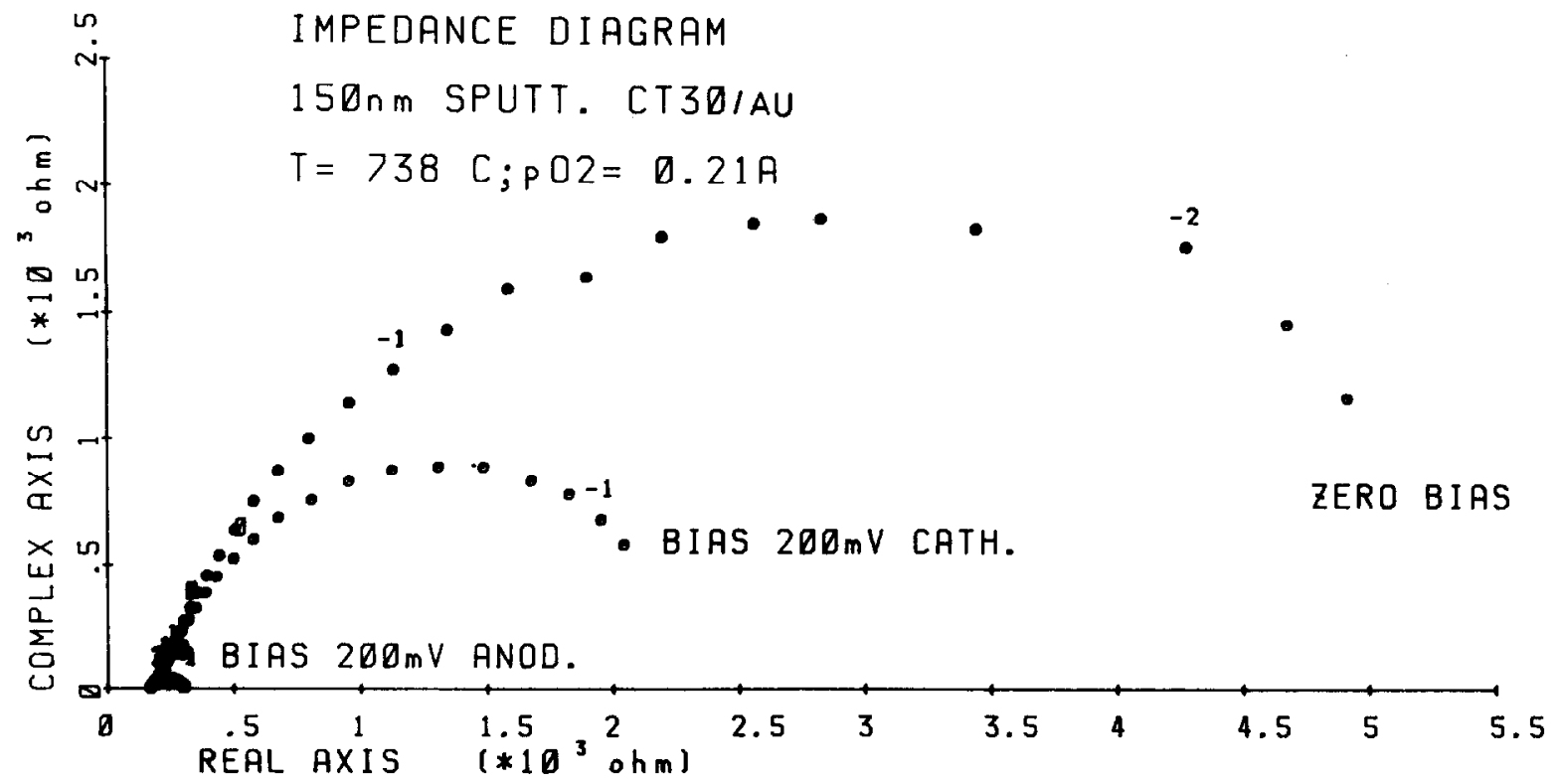

Fig.6. Polarization curves at three different values of bias conditions. 
decreasing $\mathrm{P}_{\mathrm{O}_{2}}$. Another 111ustration of this relation between $\mathrm{C}$ and $\left[\mathrm{Tb}^{4+}\right]$ is seen in the impedance diagrams for measurements under bias conditions, as is shown in Fig.6. The diagrams are dominated by the difference in electrode resistance, corresponding with the Butlervolmer behaviour, but $C$-values change too. At a negative potential of $200 \mathrm{my} \mathrm{C}=1.4 \times 10^{-4} \mathrm{~F}$, for zero bias $C=4.4 \times 10^{-4} \mathrm{~F}$ and at a positive potential of $20 \mathrm{mV}$ at the working electrode, $C=7.4 \times 10^{-4} \mathrm{~F}$. This variation of $\mathrm{C}$ with the potential results from the fact that the stolchiometry of the mixed conducting sputtered layer is changed under blas conditions.

The differences in capacitive behaviour of TGZO and CT3O is best illustrated in Fig. $3 f$, which shows the impedance diagrams for a Ptstrip at same $\mathrm{T}$ and $\mathrm{P}_{2}$. Although the polarization arcs are quite similar ( $R$, values are almost equal), the capacitive values vary over 3 decades, indicated by the numbers along the polarization arcs. The large capacitive values for CT30 and TGZ100 lead to relatively long relaxation times for the electrode reaction. This can be a severe problem in the use of these materials in sensors, which have to show short response times on changing $\mathrm{P}_{2}$.

\subsection{High frequency electrode polarization}

A stralght line was observed at the high frequency part of the electrode polarization arcs, which extends over several decades of frequency and the angle of which with the $Z^{\prime}-$ axis is between 35 and $55^{\circ}$. This implies that a diffusion process is most likely to cause the polarization effect. The fact that the behavlour is $\mathrm{P}_{\mathrm{O}_{2}}$ independent sugges ts that it concerns a bulk diffusion process. In the case of CT3O and TGZ100 both electronic and ionic charges could be responsible for the diffusion process. The activation enthalpy of $35-40 \mathrm{~kJ} / \mathrm{mol}$ for $Y_{O}$ (and for $K_{W}$ when $n=0.50$ ) can be related to the activation of the product $C / D$ from equation [4]. Assuming $C_{0}$ to be independent of $T$ to first approximation, the diffusion is activated $w i$ th an activation enthalpy of $70-80 \mathrm{~kJ} / \mathrm{mol}$. This value is somewhat higher than that for electronic conductivity $(60 \mathrm{~kJ} / \mathrm{mol}$ for TGZ100 and $52 \mathrm{~kJ} / \mathrm{mol}$ for CT30, chapter VII in [11]) but lower than the values for ionic conductivity $(=125 \mathrm{~kJ} / \mathrm{mol}$ for TGZ100 and $=115$ $\mathrm{kJ} / \mathrm{mol}$ for CT30). Therefore the diffusion is thought to result from a reorganization of electronic charges within the sputtered layer in a zone adjacent to the interface with the metal, due to the applied AC signal.

In the case of TGZO the straight line behavlour was found at higher frequencies and with an activation enthalpy of $90 \mathrm{~kJ} / \mathrm{mol}$. Here reorganization of ionic charges (oxygen vacancies) can be considered, since the activation enthalpy for oxygen ion conductivity in TGZO is in the same order. Further experiments are needed to elucidate the actual diffusion mechanism.

\section{CONCLUSIONS}

1. Results of complex Impedance analysis for small signal frequency dispersion experiments on the electrochemical oxygen gas reaction show the same trends in the polarization behaviour as was concluded from current-overvol tage experiments (see part I). Sputtered layers on top of a solid electrolyte phase improve the oxygen electrode reaction compared to a bare specimen. The largest improvement is found for the ionic conductor TGZO, and lesser improvements for the p-type mixed conductors CT 30 and TGZ100.

2. High electrode capacitances were found in the case of mixed conducting surface layers. The minimum value of the specific capacitance is $700 \mathrm{~F} / \mathrm{m}^{2}$, taken into account the fact that about $10 \%$ of the geometrical strip surface is in contact with the oxide surface. This $C$-vaiue is 1 arger than values usually found for double layer capacitances and points to pseudo-capacitances, arising from injection of electronic charges in the mixed conducting surface layer. Because of these high C-values large electrode relaxation times are found, which implies long response times in sensor applications.

3.1- $n$ curves follow a Butler-Volmer type of equation. Experimental values of $i_{0}$ and $a$ are changed from their true Faradalc values by diffusion phenomena. Most probably a diffusion process is rate controlling the charge transfer process. Surface diffusion along the solid electrolyte or sputtered layer phase can explain the difference in experimental results for different materials.

\section{ACKNOWLEDGEMENT}

Mr. H. Kruidhof and Mr. B. van Dongen are acknowledged for their experimental assistance. This investigation was supported by the Netherlands Foundation for Chemical Research (SON) with the financial aid from the Netherlands Organization for the Advancement of Pure Research (zWO). 


\section{REFERENCES}

(1) M.J. Verkerk, M.W.J. Hammink, A.J. Burggraaf, J.Elect rochem.Soc. 130 (1983) 70 and M.J. Verkerk, A.J. Burggraaf, ibidem 130 (1983) 78 .

(2) A.J.A. Winnubst, A.H.A. Scharenborg, A.J. Burggraaf, Solid State Ionics 14 (1984) 319.

(3) B.A. Boukamp, accepted for publication in Solid State Ionics.

(4) G.H.J. Broers, M. Schenke, Proc.Int.Conf. on Fuel Cells, (Akademie Verlag, Dresden, 1967), p.299.

H.T. Cahen, Ph.D. Thesis, State University Utrecht, The Netherlands (1980).

(5) M. Kleitz, J.H. Kennedy, in Fast Ion Transport in Solids ed. Vashlshta, Mundy, Shenoy (Elsevier, North Holland, 1979) p. 185 .

(6) H.J de Bruin and A.D. Franklin, J.Electroanal.Chem. 118 (1981) 405.
(7) W. Scheider J.Phys.Chem. 79 (1975) 2.

(8) H. Okamoto, G. Kawamura, T. Kudo, Electrochimica Acta 28 (1983) 379.

(9) B.C. Nguyen, D.M. Mason, L.M.Rincon-Rubio, submitted to J.Electrochem.Soc.

(10) D.Y. Wang, A.S. Nowick, J.Electrochem.Soc. 126 (1979) 1155, 1 bi dem 126 (1979) 1166 and 128 (1981) 55.

(11) M.P. van Dijk, Ph.D. Thesis, Twente University of Technology, Enschede, The Netherlands (1985).

(12) J.H.H. ter Maat, M.P. van Dijk, G.Roelofs, H. Bosch, G.M.H. van der Velde, P.J. Gellings and A.J. Burggraaf, Mat.Res. Bull. 19 (1984) 1271

(13) M.P. van Dijk, K.J. de Vries and A.J. Burggraaf, Solid State Ionics 16 (1985) 211. 